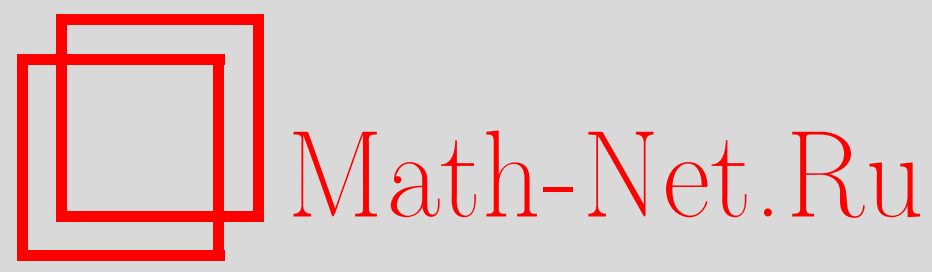

М. Е. Степанцов, Модель информационного противоборства на основе клеточного автомата, Матем. моделирование, 2020, том 32, номер 7, 47-58

DOI: https://doi.org/10.20948/mm-2020-07-03

Использование Общероссийского математического портала Math-Net.Ru подразумевает, что вы прочитали и согласны с пользовательским соглашением http://www.mathnet.ru/rus/agreement

Параметры загрузки:

IP : 3.89 .185 .249

26 апреля 2023 г., 13:03:27 


\title{
МОДЕЛЬ ИНФОРМАЦИОННОГО ПРОТИВОБОРСТВА НА ОСНОВЕ КЛЕТОЧНОГО АВТОМАТА
}

\author{
(C) 2020 2. M.E. Степанцов
}

Институт прикладной математики им. М.В. Келдыша РАН mews@yandex.ru

Работа выполнена при поддержке Российского фонда фундаментальных исследований, проекты 18-01-00619-а, 18-01-00551-а и 19-010-00423-а.

DOI: $10.20948 / \mathrm{mm}-2020-07-03$

Рассмотрены непрерывные модели информационного противоборства, основанные на традиционной нейрологической схеме. На их основе с использованием метода замены дифференциальных соотношений клеточным автоматом разработан дискретный вариант модели информационного противоборства. С ее помощью проведено моделирование агитационной кампании двух партий, на основе предложенной модели построена имитационная система, при помощи которой проведен ряд вычислительных экспериментов. В рамках этих экспериментов показано, что макродинамика новой модели соответствует макродинамике исходной, при том что дискретная модель обладает более широкой областью применимости. Для некоторых задач противоборства двух партий в рамках агитационной кампании получены результаты, аналогичные тем, которые дает непрерывная модель. Дискретная модель позволила исследовать задачу оптимального использования одной из сторон однократной дестабилизации хода агитационной кампании. В рамках этого исследования были получены оригинальные результаты, в частности - наличие критического значения коэффициента влияния общественного мнения на мнение индивида, определяющего, в какой период времени одной из сторон выгоднее повышать уровень интенсивности своей пропаганды.

Ключевые слова: математическое моделирование, имитационное моделирование, клеточные автоматы, информационное противоборство.

\section{CELLULAR AUTOMATON BASED MODEL OF INFORMATION WARFARE}

\section{M.E. Stepantsov}

Keldysh Institute of Applied Mathematics RAS

This paper considers continuous models of information confrontation based on the traditional neurological scheme. Using the method of substituting differential equations by 
cellular automata we propose a discrete version of the information warfare model. This model is used to simulate a propaganda campaign by two parties and to carry out a number of computational experiments. It is shown that the macrodynamics of the new model corresponds to one of the original, while the discrete model has a wider range of applicability. For some problems of two-party confrontation results similar to those of the continuous model were obtained. The proposed discrete model allows a study o the problem of optimal single destabilization of the campaign. This study yielded with original results, such as existence of a critical value of the public opinion influence rate, which determines the period of time profitable for increasing the level of propaganda.

Key words: mathematical modeling, simulation, cellular automata, information warfare

\section{1. Введение}

В настоящее время проблемы информационного противоборства переходят из области чистых информационных технологий в сферы политики, экономики и безопасности, и это уже не позволяет решать их качественно, получая лишь некоторые умозрительные результаты. Задачи в области информационной безопасности теперь требуют точного количественного подхода и, следовательно, развивается математический аппарат, позволяющий такой подход осуществить.

Одним из важнейших инструментов анализа информационного противоборства является математическое моделирование. С его помощью возможно не только исследовать текущее состояние социума в аспекте восприятия им информации, но также прогнозировать ход этих процессов и осуществлять вычислительные эксперименты, рассматривая различные сценарии событий и возможные варианты решения стоящих в рамках информационного противоборства задач.

Простейшие математические модели информационного воздействия на общество, а именно - модели распространения слухов, были предложены еще в шестидесятые годы прошлого века [1]. Однако в моделях этого класса (см., например, раннюю работу [2], а также современные публикации [3, 4]), рассматривается исключительно однородное общество, не учитываются возможности влияния СМИ и не рассматриваются такие аспекты принятия индивидом информации как влияние референтной группы и интериоризация взглядов индивида, полученных им извне.

В ряде более поздних работ предлагались модели информационного противоборства, избавленные от ряда перечисленных недостатков. Так, в моделях, основанных на подходе, предложенном в [5], восприимчивость индивида к информации не устанавливается в качестве постоянной, а зависит от интенсивности пропаганды [6], учитывается влияние СМИ, в том числе 
возможный неполный охват аудитории и возможность утраты индивидом интереса к информации [7].

Надо отметить, что во всех упомянутых работах состояние взглядов индивидов на проблемы, охватываемые пропагандой, рассматривалось в терминах непрерывных функций и дифференциальных уравнений. Такой подход основывается на классических методах математического моделирования, которые хорошо известны и исследованы, поэтому зачастую могут быть успешно применяемы. С другой стороны, непрерывный характер зависимостей несколько ограничивает область применимости моделей и не всегда корректен методологически, поскольку моделируемая реальность является существенно дискретной. Также важно отметить, что наибольшее влияние на мнение индивида оказывают малые группы, между тем как в непрерывной модели рассматривается влияние общества в целом, в то время как дискретная модель на основе клеточного автомата позволяет рассмотреть влияние малой референтной группы на формирование мнения индивида.

В данной работе рассмотрим модели информационного противоборства [8,9], основанные на традиционной нейрологической схеме [10].

В рамках классического подхода был исследован простейший случай пропаганды $[8,11]$, в котором речь шла о выборе индивидами одной из двух позиций по некоторому вопросу, например, какую из партий - L или $\mathrm{R}-$ поддержать на выборах.

Модель, предложенная в [8], имеет вид интегро-дифференциального уравнения

$$
\frac{d \psi}{d t}=A\left(C\left(2 \int_{-\psi(t)}^{+\infty} N(\varphi) d \varphi-N_{0}\right)+b_{R}-b_{L}\right)-a \psi
$$

с начальным условием, задаваемым в виде

$$
L(0)=\int_{-\infty}^{-\psi(0)} N(\varphi) d \varphi .
$$

Здесь функция $\psi(t)$ имеет смысл сдвига предпочтений индивидов под влиянием пропаганды, определяя численность сторонников партий L и R как

$$
\begin{aligned}
& L(t)=\int_{-\infty}^{-\psi(t)} N(\varphi) d \varphi, \\
& R(t)=\int_{-\psi(t)}^{+\infty} N(\varphi) d \varphi,
\end{aligned}
$$


т.е. функция $N(\varphi)$ задает распределение отношения индивидов к альтернативам L и R, a

$$
N_{0}=\int_{-\infty}^{+\infty} N(\varphi) d \varphi
$$

представляет собой численность социума. Параметры $b_{R}, b_{L}, C, A$ и $a$ характеризуют, соответственно, влияние пропаганды партий $\mathrm{R}$ и L, общественного мнения, способность индивидов менять свое мнение и «затухание» таких изменений.

Распределение $N(\varphi)$ характеризует распределение в обществе взглядов на проблему выбора между альтернативами. Нормальный закон этого распределения с центром в нуле соответствует консолидированному обществу, а сумма двух функций нормального распределения с симметричными относительно нуля центрами описывает поляризованное по данному вопросу общество [11].

Хотя точное решение задачи (1), (2) в общем виде не получено, соответствующая стационарная задача исследована в [8]. Однако рассмотренная непрерывная модель не всегда может быть удачно модифицирована для учета дополнительных социальных или психологических аспектов. Так, в ее рамках трудно учесть возможную различную восприимчивость индивидов к пропаганде, особое влияние мнения референтной для данного индивида группы и вообще дисперсию любых характеристик индивидов, кроме, собственно, их априорных отношений к альтернативам.

Одним из способов преодоления указанных трудностей является замена непрерывной модели клеточным автоматом, макродинамика которого будет соответствовать динамике решений приведенного выше интегро-дифференциального уравнения. Такой подход уже применялся автором к модели А.П. Михайлова «Власть-общество» и ее модификациям [12]. Он является с некоторой точки зрения более адекватным и потому, что речь идет о моделировании дискретных явлений при помощи дискретных математических объектов без промежуточного перехода к непрерывному рассмотрению.

Мы ограничимся построением такой дискретной модели на основе клеточного автомата, которая бы описывала ту же реальность, что и исходная непрерывная модель информационного противоборства, демонстрировала бы такую же макродинамику и давала возможность повторить результаты, полученные при помощи непрерывной модели. Модификации, позволяющие расширить область применимости модели, будут предметом исследований, планируемых в дальнейшем. 


\section{2. Построение модели}

В качестве поля предлагаемого клеточного автомата возьмем классическую ортогональную решетку, в каждом из узлов которой разместим конечный автомат, описывающий текущие взгляды отдельного индивида. В рамках традиционной для клеточных автоматов терминологии будем называть его клеткой, имеющей три возможных состояния: поддержка альтернативы $\mathrm{L}(-1)$, поддержка альтернативы R (1) и не определившиеся взгляды (0). Также припишем каждой клетке в качестве параметра состояние взглядов индивида без воздействия пропаганды, которое также может принимать одно из трех перечисленных значений, но является неизменным - в отличие о состояния клетки, описывающего текущие взгляды индивида.

В рамках этого подхода для данной модели вместо непрерывного распределения индивидов по взглядам на предлагаемые альтернативы будет использовано их дискретное распределение по трем возможным точкам зрения. В качестве величины, аналогичной (но не равной в точности) переменной $\psi$ будет выступать выражение

$$
\psi^{*}=\frac{R(t)-L(t)}{N_{0}} .
$$

Тогда динамика этой величины задается следующим образом:

$$
\frac{d \psi^{*}}{d t}=\frac{d R(t) / d t-d L(t) / d t}{N_{0}} .
$$

Дифференцируя (3) и (4), получаем

$$
\frac{d \psi^{*}}{d t}=2 \frac{d \psi}{d t} \frac{N(\psi)}{N_{0}}
$$

Таким образом, динамика величины $\psi^{*}$ не совпадает в точности с динамикой $\psi$, но, благодаря положительности множителя $N(\psi) / N_{0}$, имеет ту же направленность.

Соотношение (5) можно использовать для проверки соответствия макродинамики дискретной и непрерывной моделей, но следует заметить, что большая часть результатов в $[8,11]$ получена в терминах динамики численности сторонников альтернатив, т.е. сравнение моделей уместно проводить именно по этим показателям.

Итак, можно легко «перевести на язык клеточного автомата» все члены правой части уравнения (1), кроме интегрального. Что же касается интегрального слагаемого, то очень удачным оказывается тот факт, что оно 
представляет собой не что иное, как разность количеств сторонников двух альтернатив $R(t)-L(t)$. Эту величину мы можем получить прямым подсчетом по полю клеточного автомата. Таким образом, на каждом шаге по времени в предлагаемом клеточном автомате к каждой клетке применяются последовательно три алгоритма.

Алгоритм А: влияние прямой пропаганды.

if $\Delta>0$ then

if Center $=0$ and $r<\Delta$ then Center $=1$

if Center $=-1$ and $r<\Delta$ then Center $=0$

end if

if $\Delta<0$ then

if Center $=0$ and $r<-\Delta$ then Center $=-1$

if Center $=1$ and $r<-\Delta$ then Center $=0$

end if

Здесь $\Delta=A^{*}\left(b_{R}-b_{L}\right)$ - параметр, отвечающий за суммарное влияние прямой пропаганды в пользу каждой из альтернатив, $r$ - случайное число, равномерно распределенное на промежутке [0;1], Center - стандартное обозначение состояния самой (в отличие от ее соседей) рассматриваемой клетки поля клеточного автомата.

Алгоритм Б: затухание изменений точки зрения, вызванных пропагандой. if not $z=$ Center then

if $r<a^{*}$ then

if Center $>z$ then Center $=$ Center -1

if Center $<z$ then Center $=$ Center +1

end if

end if

Здесь $a^{*}$ - параметр, отвечающий за затухание влияния пропаганды, $z$ параметр, устанавливающий начальное состояние клетки (априорное отношение индивида к альтернативам), заданный для каждой клетки.

Алгоритм В: влияние общественного мнения.

if $\psi^{*}>0$ then

if Center $<1$ and $r<c^{*} \psi^{*}$ then Center $=$ Center +1

end if

if $\psi^{*}<0$ then

if Center $>-1$ and $r<-c^{*} \psi^{*}$ then Center $=$ Center -1

end if

Здесь $\psi^{*}$ - введенная ранее величина, характеризующая превышение числа сторонников альтернативы $\mathrm{R}$ над числом сторонников альтернативы $\mathrm{L}, c^{*}$ - параметр, описывающий влияние общественного мнения. 


\section{3. Вычислительные эксперименты}

3.1. Проверка адекватности модели. Предложенный клеточный автомат, как показано выше, при соответствующем подборе коэффициентов должен порождать такую же макродинамику, как и исходная непрерывная модель. Для проверки этого утверждения был проведен ряд вычислительных экспериментов. В них использовался клеточный автомат с размерами поля $20 \times 20$, содержащий 400 клеток. В каждом эксперименте начальное распределение мнений совпадало с полем значений априорных мнений $z$, т.е. предполагалось, что в начальный момент времени $\psi^{*}=0$. В качестве числа не определившихся индивидов было взято нормированное соответствующим образом значение непрерывной функции распределения при $\varphi=0$, а в качестве чисел сторонников двух альтернатив - значения этой функции в точках перегиба для консолидированного общества и ее значения в максимумах для поляризованного. Клетки, моделирующие сторонников каждой из альтернатив и не определившихся, были размещены на поле клеточного автомата случайным образом. Точные количества априорных сторонников каждого из вариантов для $N_{0}=400$ приведены в табл.1.

Таблица 1. Дискретное распределение априорных предпочтений для поля размером 20×20.

\begin{tabular}{|l|c|c|c|}
\hline \multirow{2}{*}{ Тип общества } & \multicolumn{3}{|c|}{ Количество индивидов } \\
\cline { 2 - 4 } & Сторонники L & Неопределившиеся & Сторонники R \\
\hline Консолидированное & 110 & 180 & 110 \\
\hline Поляризованное & 150 & 100 & 150 \\
\hline
\end{tabular}

Прежде всего, в рамках нескольких сотен экспериментов были получены зависимости $\psi^{*}(t)$ при различных значениях параметров. В каждом случае эта величина стремилась к стационарному значению, следовательно, как и в [11], формировались стационарные значения численности сторонников каждой из альтернатив. Типичный пример такой зависимости представлен на рис.1.

Поскольку, как и в случае непрерывной модели, имеет место выход динамики системы на стационарное решение, оказывается возможным, как это было сделано в [11], исследовать функцию отклика на пропаганду. Эта функция представляет собой зависимость стационарного значения численности сторонников одной из альтернатив (например, R) от интенсивности пропаганды в поддержку этой альтернативы.

В экспериментах были использованы значения параметров $b_{L}=10$, $c^{*}=0.1, a^{*}=0.1, A^{*}=0.1$ и изучалась зависимость стационарного значения $\mathrm{R}$ от параметра $b_{R}$, который менялся в интервале от 0 до 26 . Как и для не- 
прерывной модели в [11], в случае консолидированного общества эта зависимость представляла собой функцию, выпуклую вверх (рис.2), а при поляризованном обществе - функцию с S-образным графиком (рис.3).

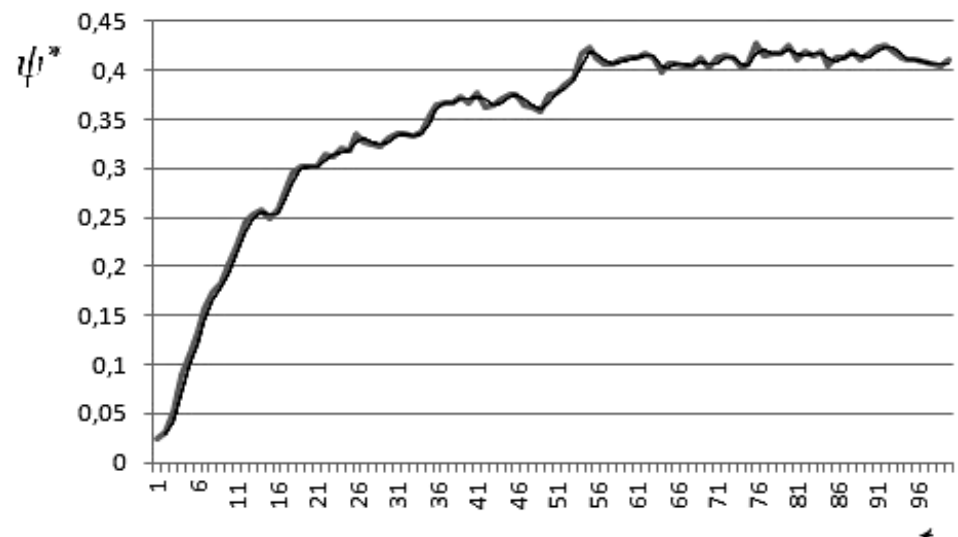

Рис.1. Формирование стационарных значений численностей сторонников альтернатив.

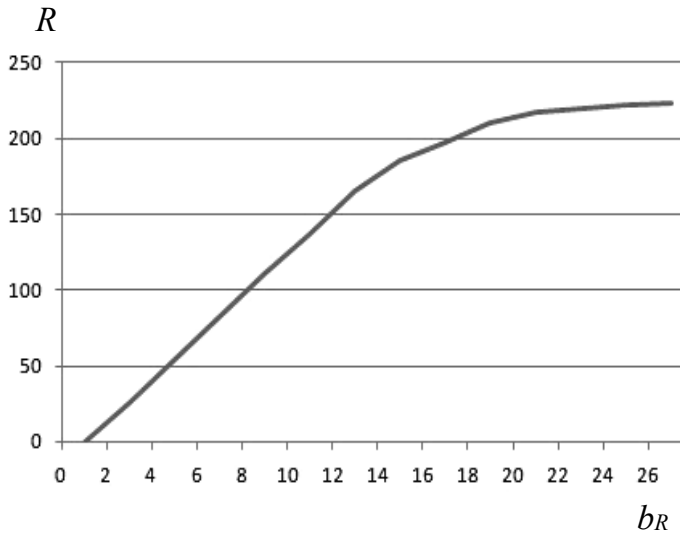

Рис.2. Функция отклика для случая консолидированного общества.

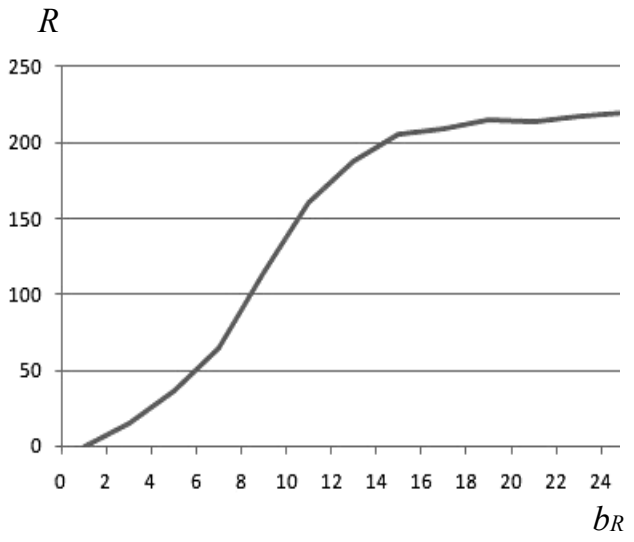

Рис.3. Функция отклика для случая поляризованного общества.

Таким образом, предложенная в работе дискретная модель на основе клеточного автомата имеет макродинамику, соответствующую макродинамике исходной непрерывной модели и позволяет получить результаты, аналогичные тем, которые были получены при помощи непрерывной модели, что свидетельствует об адекватности предлагаемой модели.

Учтем, что непрерывная модель не учитывает то, что наибольшее влияние на мнение индивида оказывают малые группы, в ней рассматривается влияние общества в целом. Предлагаемая модель позволяет рассмотреть это влияние как воздействие окрестности клетки на ее состояние, что является 
отличительной чертой клеточного автомата. Кроме того, в исходной модели не рассматривается процесс интериоризации индивидом внешних представлений, что легко можно осуществить в клеточно-автоматной модели путем задания правил изменения параметров $z$, описывающих внутренние убеждения индивидов. В тех случаях, когда перечисленные аспекты могут оказаться важными для понимания динамики изучаемой системы, представляется уместным использовать дискретную клеточно-автоматную модель.

\section{2. Исследование оптимального распределения интенсивности про-} паганды при одноразовой дестабилизации. Предлагаемая дискретная модель позволяет, помимо другого расширения области применимости, рассматривать зависимости параметров $b_{R}$ и $b_{L}$ от времени, носящие произвольный характер, в то время как в базовой классической модели эти параметры предполагаются постоянными, а в рамках ее исследований рассматривались их зависимости от времени, имеющие вид отдельных непрерывных дифференцируемых функций.

В частности, в [13] речь шла о периодической дестабилизации информационного противоборства, т.е. рассматривалась возможность одной из партий $(\mathrm{R})$ осуществлять периодическое изменение интенсивности своей пропаганды.

Представляет интерес рассмотреть возможность одной из сторон однократно усилить пропаганду на некоторый период времени. В рамках данной работы было проведено исследование зависимости итогового результата для альтернативы $\mathrm{R}$ от соотношения параметров $a^{*}$ (коэффициента влияния общественного мнения) и $c^{*}$ (характеризующего затухание изменений мнений индивидов, произошедших под влиянием пропаганды).

В экспериментах этой серии было использовано то же поле клеточного автомата с такими же распределениями априорных взглядов, как и в первой, и значения параметров $b_{L}=10, A^{*}=0.1$. Рассматривался временной промежуток длительностью 50 шагов по времени, что можно рассмотреть как 50 дней ведения пропагандистской кампании. В течение любого промежутка длительностью 5 дней партия $\mathrm{R}$ имела возможность повысить уровень своей пропаганды $b_{R}$ до 19 при том, что в остальные дни он должен был оставаться равным 9, что делало суммарные интенсивности пропаганды обеих сторон равными. Такие вычислительные эксперименты проводились для консолидированного и поляризованного типов обществ при различных значениях параметров $a^{*}$ и $c^{*}$. При этом, результаты экспериментов для обоих типов обществ практически не отличались между собой.

Оказалось, что, если зафиксировать значение $c^{*}$, то при низких значе- 
ниях $a^{*}$ оптимальный результат партии $\mathrm{R}$ получается при повышении интенсивности пропаганды в последние 5 дней, а при высоких - в первые 5 дней. Это был вполне ожидаемый из общих соображений эффект.

Следует отметить, что ни в одном эксперименте результат R не был оптимальным при каком-либо ином расположении отрезка повышенной интенсивности пропаганды на временной шкале. При этом, естественно, при каждом наборе значений других параметров существует некоторое критическое значение $a_{0}^{*}$ такое, что при более низких значениях $a^{*}$ оптимальным является повышение интенсивности в конце периода, а при более высоких в начале. Пример результатов моделирования пропагандистской кампании при $c^{*}=0.1$ приведен в табл.2. Здесь $a_{0}^{*} \approx 0.16$.

В ходе вычислительных экспериментов выяснилось, что в некоторой небольшой окрестности точки $a_{0}^{*}$ дестабилизация уровня пропаганды приводит к проигрышу альтернативы $\mathrm{R}$ по сравнению со стратегией равномерной интенсивности, в то время как при достаточно больших или достаточно малых значениях $a^{*}$ оптимальный результат $\mathrm{R}$ оказывается лучше, чем при использовании стратегии равномерной интенсивности.

Таблица 2. Значения $\psi^{*}$ по результатам моделирования 50-дневной пропагандистской кампании. Выделены оптимальные для партии $R$ результаты.

\begin{tabular}{|l|l|l|l|}
\hline \multirow{2}{*}{$a^{*}$} & \multicolumn{3}{|l|}{ Повышение интенсивности пропаганды } \\
\cline { 2 - 4 } & в начале & в середине & в конце \\
\hline 0.1 & -0.325 & -0.11 & $\mathbf{0 . 0 5 7 5}$ \\
\hline 0.15 & -0.1525 & -0.08 & $\mathbf{0 . 0 2 7 5}$ \\
\hline 0.16 & -0.051 & -0.055 & $\mathbf{- 0 . 0 4 5}$ \\
\hline 0.2 & $\mathbf{0 . 0 3 7 5}$ & -0.014 & -0.065 \\
\hline 0.25 & $\mathbf{0 . 3 8}$ & 0.01 & -0.36 \\
\hline 0.5 & $\mathbf{0 . 9 1 2 5}$ & 0.35 & -0.8925 \\
\hline
\end{tabular}

Графики типичных зависимостей результатов партий $\mathrm{R}$ и $\mathrm{L}$ от значения $a^{*}$ приведены на рис.4.

\section{4. Заключение}

Таким образом. с использованием метода замены дифференциальных зависимостей клеточным автоматом, была построена дискретная модель информационного противоборства. Она была реализована в виде программы в среде VBA и с ее помощью были осуществлены две серии вычислительных экспериментов.

Первая серия имела целью проверку адекватности модели в смысле соответствия ее макродинамики макродинамике непрерывной модели, и таковая цель была достигнута. 


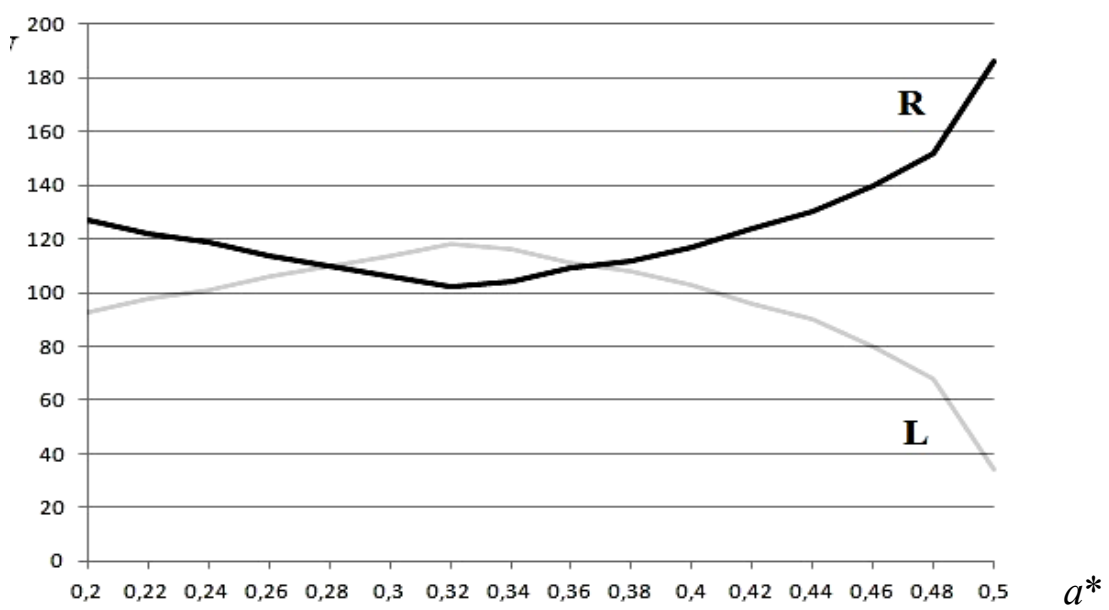

Рис.4. Численности сторонников партий $\mathrm{R}$ и $\mathrm{L}$ при наилучшем для $\mathrm{R}$ результате пропагандистской кампании при различных значениях $a^{*}$.

Вторая серия была посвящена рассмотрению проблемы, которая не вполне могла быть изучена при помощи непрерывной модели, а именно влияния однократного непериодического возмущения интенсивности пропаганды на ход кампании. В ходе этого исследования были получены следующие результаты.

1. Оптимальным управлением для партии $\mathrm{R}$ является повышение уровня пропаганды в начале агитационного периода при больших по сравнению с $c^{*}$ значениях $a^{*}$, и в конце этого периода при малых. Выбор промежуточного момента времени для повышения интенсивности пропаганды не является оптимальным ни в каком случае.

2. Для каждого значения $c^{*}$ существует критическое значение $a_{0}^{*}$, такое, что при меньших значениях выгоднее повышать интенсивность пропаганды в конце периода агитации, а при больших - в его начале.

3. В небольшой окрестности этого значения оптимальный результат для партии $\mathrm{R}$ оказывается хуже результата в отсутствие дестабилизации, для остальных случаев использование дестабилизации приводит к выигрышу альтернативы R.

4. Полученные закономерности имеют место как для консолидированного, так и для поляризованного общества. Можно предположить, что они вообще слабо зависят от априорного распределения мнений.

\section{СПИСОК ЛИТЕРАТУРЫ}

1. D.J. Daley, D.G. Kendall. Stochastic rumors // Journal of the Institute of Mathematics and its Applications, 1964, v.1, p.42-55. 
2. D.P. Maki, M. Thompson. Mathematical Models and Applications. - Prentice-Hall, Englewood Cliffs, NJ, USA, 1973.

3. Liang'an Huo, Peiqing Huang, Chun-Xiang Guo. Analyzing the Dynamics of a Rumor Transmission Model with Incubation // Discrete Dynamics in Nature and Society, 2012, v.2012, Article ID 328151, 21 p.

4. R. Isea, R. Mayo-García. Mathematical analysis of the spreading of a rumor among different subgroups of spreaders // Pure and Applied Mathematics Letters, 2015, v.2015, p.50-54.

5. А.А. Самарский, А.П. Михайлов. Математическое моделирование. Идеи, методы, примеры. - М.: Физматлит, 1997, 320 с.;

A.A. Samarskii, A.P. Mikhailov/ Matematicheskoe modelirovanie. Idei, metody, primery. M.: Fizmatlit, 1997, $320 \mathrm{s.}$

6. А.П. Михайлов, Н.А. Маревцева. Модели информационной борьбы // Математическое моделирование, 2011, т.23, №10, с.19-32;

A.P. Mikhailov, N.A. Marevtseva. Models of information warfare // MM\&CS, 2012, v.4, №3, p.251-259.

7. А.П. Михайлов, А.П. Петров. Основные направления математического моделирования информационного противоборства в социуме // Представительная Власть - ХХІ век, 2019, №5-6, c.36-46;

A.P. Mikhailov, A.P. Petrov. Osnovnye napravleniia matematicheskogo modelirovaniia informatsionnogo protivoborstva v sotsiume // Predstavitelnaia vlast - XXI vek, 2019, №5-6, p.36-46.

8. А.П. Петров, А.И. Маслов, Н.А. Цаплин. Моделирование выбора позиций индивидами при информационном противоборстве в социуме // Математическое моделирование, 2015, т.27, №12, с.137-148;

A.P. Petrov, A.I. Maslov, N.A. Tsaplin. Modeling position selection by individuals during information warfare in society // MM\&CS, 2016, v.8, №4, p.401-408.

9. A.P. Petrov, S.A. Lebedev. Online Political Flashmob: the Case of 632305222316434 // Computational mathematics and information technologies, 2019, №1, p.17-28.

10. N. Rashevsky. Mathematical biophysics: physico-mathematical foundations of biology. Univ. of Chicago: Chicago Press, 1938.

11. О.Г. Прончева, А.П. Петров. Функция отклика на пропаганду в консолидированных и поляризованных обществах // Информационные войны, 2018, №3 (47), с.50-53;

O.G. Proncheva, A.P. Petrov. Funktsiia otklika na propagandu v konsolidirovannykh i poliarizovannykh obshchestvakh // Informatsionnye voiny, 2018, №3 (47), s.50-53.

12. M.E. Степанцов. Моделирование системы «власть-общество-экономика» с элементами коррупции на основе клеточных автоматов // Математическое моделирование, 2017, т.29, №9, c.101-109;

M.E. Stepantsov. Simulation of the "Power-Society-Economics" System with Elements of Corruption Based on Cellular Automata // MM\&CS, 2018, v.10, №2, p.249-254.

13. А.П. Михайлов, А.П. Петров, О.Г. Прончева. Модель информационного противоборства в социуме с кусочно-постоянной функцией дестабилизирующего воздействия // Математическое моделирование, 2018, т.30, №7, с.47-60;

A.P. Mikhailov, A.P. Petrov, O.G. Proncheva. A Model of Information Warfare in a Society with a Piecewise Constant Function of the Destabilizing Impact // MM\&CS, 2019, v.11, №2, p.190-197.

Поступила в редакцию 07.11.2019 После доработки 07.11.2019

Принята к публикации 23.12.2019 